\title{
Effect of Tai Chi and Stepping Exercise for Balance Training in Geriatrics: Randomized Control Trial
}

\author{
Gaurav C. Mhaske ${ }^{1}$, Darshana Fursule ${ }^{2}$, Anagha Khairnar ${ }^{3}$ \\ ${ }^{1}$ Assistant Professor, MGM Institute of Physiotherapy, Aurangabad. \\ ${ }^{2}$ Intern, MGM Institute of Physiotherapy, Aurangabad. \\ ${ }^{3}$ Intern, MGM Institute of Physiotherapy, Aurangabad. \\ Corresponding Author: Darshana Fursule
}

\begin{abstract}
Introduction: Approximately $40 \%$ of geriatric population in India experience some form of balance difficulty. It may be due to decreased muscular strength, flexibility, impaired vision and hearing problem. There are many approaches for improving balance such as Tai Chi exercise, stepping exercise, wobble board exercises, strengthening exercises. Tai Chi is a series of slow, smooth and graceful movements for improving neuromuscular function, endurance, joint sense and cognitive function. Stepping exercise is exercise performed using stepper. It improves balance by strengthening muscles, flexibility and range of motion.

Objective: To compare the effect of Tai Chi and stepping exercise for improving balance in geriatric population.

Method: In the present study, 40 participants of age group 60-80 years with balance problem were taken. Random sampling was done in two group, group A $(n=20)$ and group $B(n=20)$ giving Tai Chi and Stepping exercise respectively. Outcome measured used were BBS, TUG, FES and POMA.

Results: In present study, Tai Chi exercise was more effective than Stepping exercise statistically proved with unpaired $\mathrm{t}$ test BBS $(\mathrm{p}=0.0001), \mathrm{TUG}(\mathrm{p}=0.0001)$, FES $(\mathrm{p}=0.0679)$ and POMA $(p=0.0039)$. When comparison was done within the groups, all outcome measures showed significant values at pre and post intervention, BBS ( $p=<0.0001$ in group A and $B$ ), TUG ( $p=<0.0001$ in group A and $B)$, FES ( $p=<0.0001$ in group A and $B)$ and POMA ( $p=<0.0001$ in group A and $B)$,

Conclusion: Tai Chi exercise showed more effect than Stepping exercise in geriatric population with balance impairments.
\end{abstract}

Keywords: Tai Chi exercise, stepping exercise, geriatric population, Balance Training in Geriatrics, balance impairments

\section{INTRODUCTION}

Ageing is the process of change in the properties of structure or system occurring over a period, either spontaneously or though deliberate action. ${ }^{1}$ It is estimated that approximately $40 \%$ of geriatric population in India experience some form of balance difficulty. Conditions associated with balance problems include dizziness, muscular weakness, impaired vision, hearing, diabetes. ${ }^{4}$

According to 2011 population census, nearly 104 million elderly persons are in India. ${ }^{2}$ The sex ratio among elderly people was as high as 1028 in 1951 but subsequently dropped to about 938 in 1971, but has finally increased again to about 972 in 2001. According to 2011 population census there are 53 million females and 51 million males in India. ${ }^{3}$

Fall are defined as an event which results in person coming to rest inadvertently on a ground or floor or other lower level. Falls are major public health problem. It is second leading cause of unintentional injury death, after road traffic accident. $31 \%$ of 

control trial.

individuals aged 65 years and older fall at least once year. The prevalence of fall in India among older adults aged 60 years and older was $14 \%$ to $53 \% .^{6}$

Balance is staying upright and steady when stationary, such as when standing or sitting, or during movement. The loss of ability to balance may be linked with a higher risk of falling, increased dependency, illness and sometimes early death. ${ }^{7}$

Tai chi exercise is the task of maintaining balance which becomes highly difficult, as evidenced by the increased frequency of falls in older adults. Tai Chi is a traditional Chinese form of exercise among older adults, especially in Asia used for improving balance and other neuromuscular functions. It's a series of slow, smooth, and graceful movements, with an emphasis on coordination of the eyes, head, body, and upper and lower extremities. Timothy $\mathrm{C}$ et.al has shown Tai Chi training and practice there was significant improvements in balance and it had beneficial effects on health. ${ }^{8}$ A. Vonkelatos, L., et al they concluded that Tai Chi appeared to reduce the falls in geriatric population. $^{14}$

Stepping represents a common means for balance recovery after a perturbation to upright posture. ${ }^{10}$ It is a form of aerobics exercise using an elevated platform. The height can be tailored to individual needs by inserting risers under the step. It helps to improve balance in elderly. It strengths the muscles which helps in maintain balance. ${ }^{11}$ Ryosuka shigematsu, et.al concluded that steeping exercise is more effective then walking in reducing fall risk factors. Hence stepping exercise is effective in balance training in geriatric population ${ }^{11}$.

Hence, this study aimed the dearth to investigate which is the enhanced the effects of Tai Chi and Stepping exercises on balance in elderly people. ${ }^{9}$ The hypothesis of the study there will be improvement in balance by Tai Chi exercise than Stepping exercise in geriatric population.

\section{METHODOLOGY}

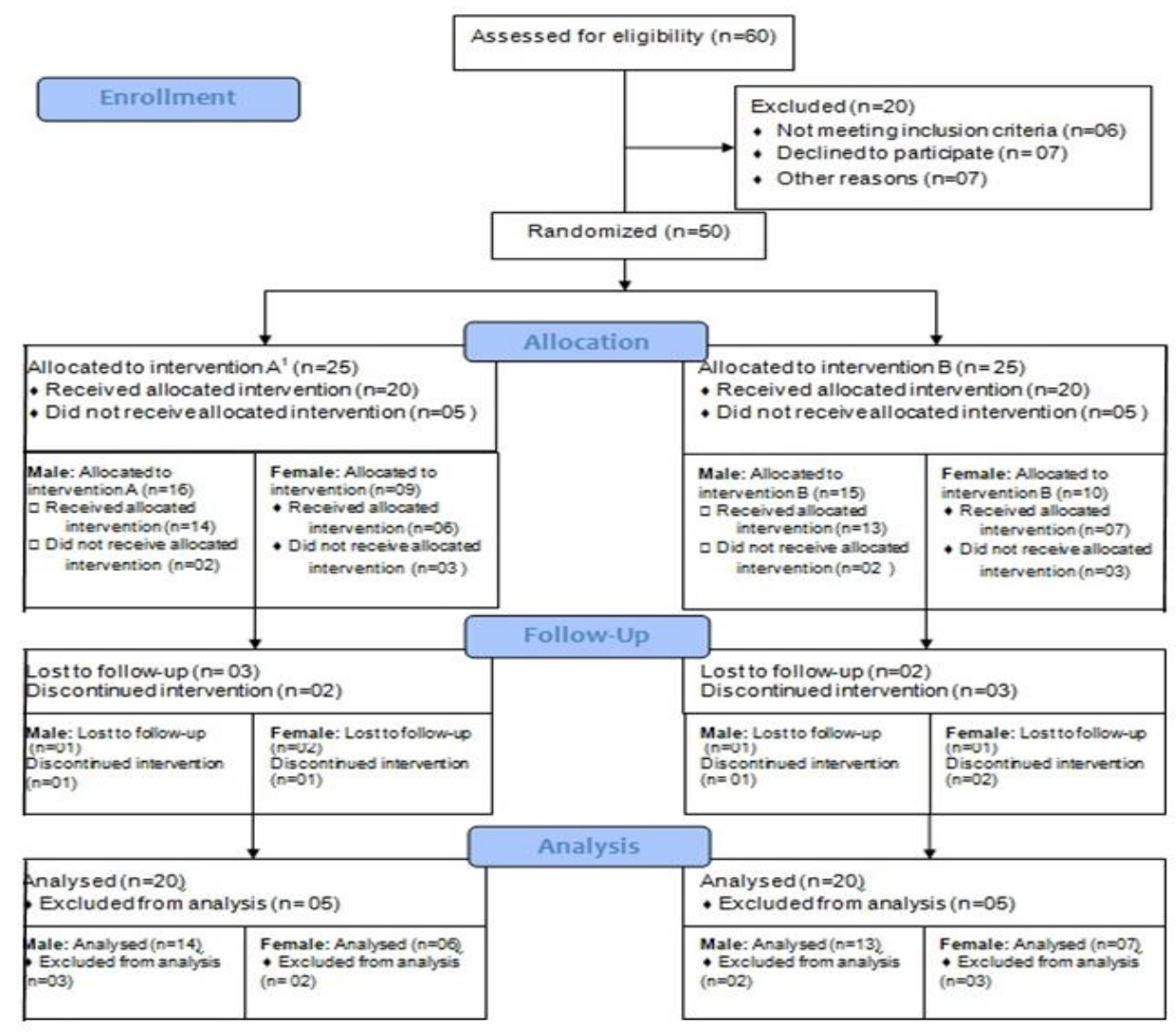



control trial.

The study design for this research is comparative study. Data was collected from MGM hospital, Aurangabad and Old age home in and Around Aurangabad City. 40 Convenience sampling were taken for this study and randomly divide into two groups by using envelope method. Inclusion criteria were: 60 to 80 years Age group, Able to walk independently, Mini mental state examination score greater than 24 and Berg Balance Scale score greater than 14. Exclusion criteria: A medical history that contained current treatment for recent fracture, uncontrolled diabetes and seizure disorder, Fainting and dizziness, and any cognitive behavioral and perceptual problems. Materials used were Non slippery surface, Countdown Timer (stop watch), Chair, Step platform and Music System.

\section{PROCEDURE:}

There was 2 group which were included in this study, Group A Tai Chi exercise and Group B Stepping exercise for balance training in geriatric population. Total number of 50 participants were divided into group A $(n=20)$ and group $B$ $(n=20)$ by envelope methods respectively. In that 40 people were recruited for this study. Pre, intermediate and post values were taken for motor function with the independence IV. level. The purpose of the study was explained, the subjects were screened based on the inclusion and exclusion criteria and examinations were done using BBS, TUG,
FES and POMA. The treatment was 5 sessions per week for 4 weeks each session for 45-60 minutes. Written consent was obtained from all the participants after their inclusion and the treatment session. ${ }^{12}$

\section{Group A (Tai Chi exercises)}

Patient received for Tai Chi training. Before training group A received training for a total of 4 weeks with 5 session per week for 45 to 60 minutes. The sessions covered theoretical background and demonstration various forms of Tai Chi exercises. $^{13}$

\section{PROCEDURE:}

A. Patient - explanation of the reason why using Tai Chi exercises and obtaining the patient's consent to the treatment before proceeding.

B. Briefing the patient about Tai Chi exercise and its benefits.

\section{Application:}

I. The first $10 \mathrm{~min}$ session was reserved for warm up.

II. Further 30 min session was reserved for various forms of Tai Chi exercises.

III. Further 5 to $10 \mathrm{~min}$ was reserved for cool down.

Patients was advised for extra 2 session in a week of Tai Chi exercises by their excluding above mentioned sessions so that proper effect can be seen.

\section{Various forms of Tai Chi exercises ${ }^{12}$}

\begin{tabular}{|l|l|}
\hline FORM & MOVEMENT DIRECTION AND NUMBER OF REPETATIONS \\
\hline 1. Commencing form & Both hands rise to shoulder level \\
\hline 2. Curving back arms(Repulse Monkey) & Right, left; 3 times each side \\
\hline $\begin{array}{l}\text { 3. Stepping sideways and moving arms (Grasp Peacock's Tail: } \\
\text { ward off, rollback, press, push) }\end{array}$ & To the left then to the right \\
\hline 4. Moving hands (wave hands like clouds) & Left side leads, 3 times \\
\hline 5. Diagonal strides (Fair lady works at shuttles) & Left, then right \\
\hline 6. Standing on one leg(Golden Cock stand on one leg) & Right, then left \\
\hline 7. Stepping and pushing (Brush knees and twist step) & Left, then right \\
\hline 8. Closing form & Both hands fall to the side, left leg drawn to the right leg. \\
\hline
\end{tabular}

\section{Group B (Stepping exercises)}

Patient received for Tai Chi training.

Before training examinations were done using BBS, TUG, FES and POMA.
Group B received treatment for 4 weeks with 5 sessions per week for 30 to 45 minutes. The sessions covered theoretical background and demonstration of 
Gaurav C. Mhaske et.al. Effect of tai chi and stepping exercise for balance training in geriatrics: randomized control trial.

standardized technique used in stepping exercises. ${ }^{16}$

\section{PROCEDURE:}

A. Patient -explanation of the reason why using stepping exercises and obtaining III. the patient's consent to the treatment before proceeding.

B. Briefing the patient about stepping exercise and its benefits.

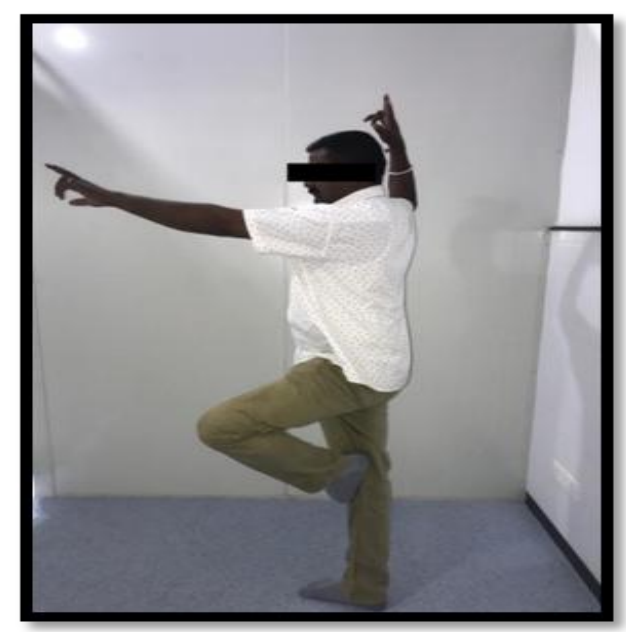

\section{Application:}

I. The first $10 \mathrm{~min}$ session was reserved for warm up.

II. Further 30 min session was reserved for various forms of Stepping exercises.

III. Further 5 to $10 \mathrm{~min}$ was reserved for cool down.

IV. Patients was advised for extra 2 session in a week of Stepping exercises by their excluding above mentioned sessions so that proper effect can be seen.

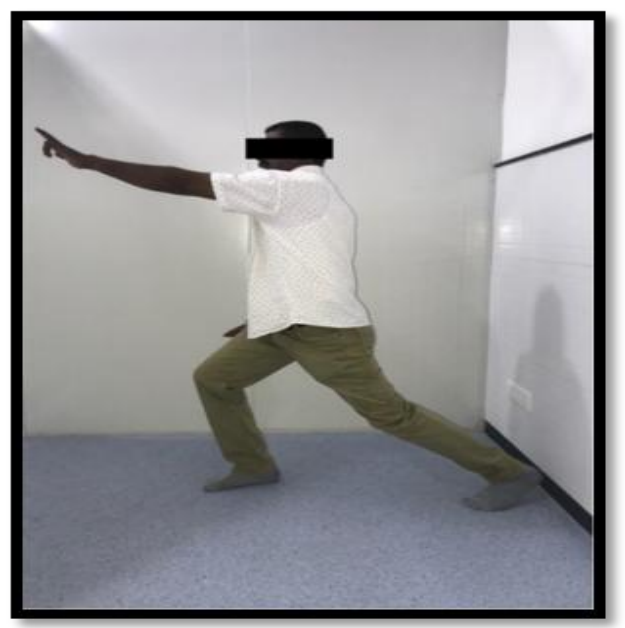

Fig: Tai Chi exercises
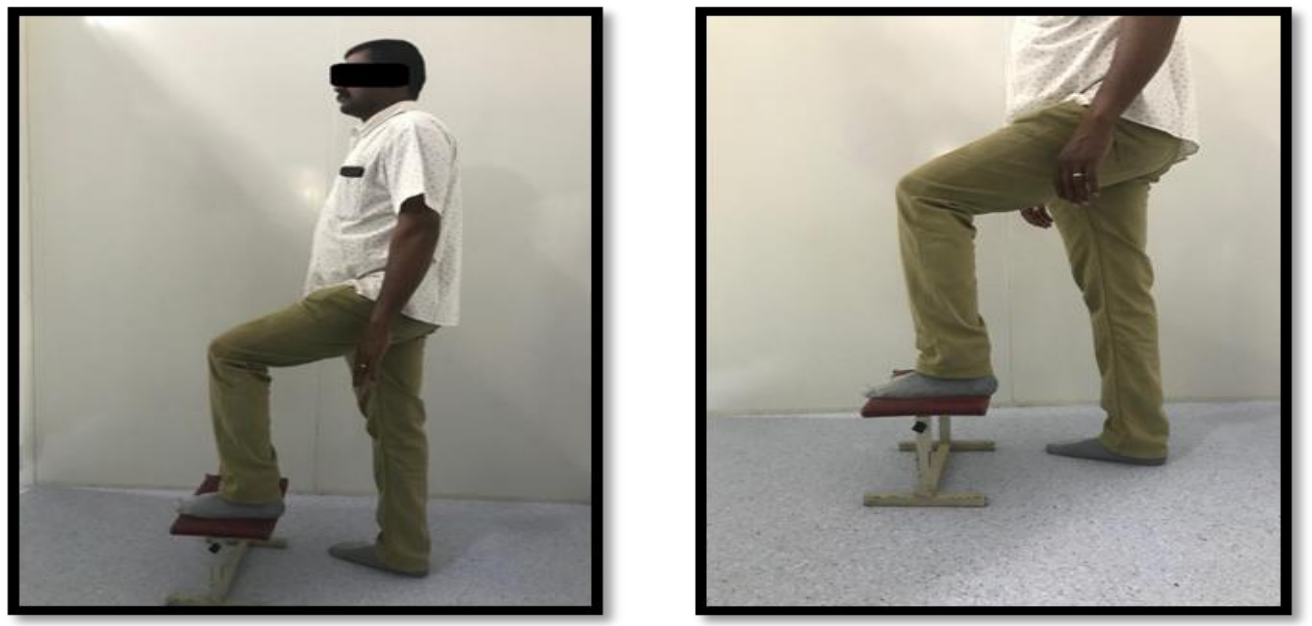

Fig no. 6: Stepping exercise

\section{OUTCOMES MEASURES USED:}

1. Berg balance scale 14 items scale 56 components designed to measure balance of older adults in clinical setting. The berg balance scale is reliable and valid measure that is used to asses characteristic in balance. Total score $=56$.
Inter-rater reliability estimated at 0.97 and intra-rater reliability estimated at $0.98 .^{21}$

2. Timed up and go test: this scale is to assess the functional mobility of the patient. Person who ever performing this test should wear the light foot wear and stand from a chair and walks for 3 meters. Timed up and go scale is having 
good reliability and validity to assess the functional mobility in old agepatient. ${ }^{22}$

3. Fall efficacy scale: this scale is used to measure fear of fall in older persons. Fall efficacy scale, on scale from 1 to 10 , with 1 being very confident and 10 being not confident at all. A total score of greater than 70 indicates that the person has fear of falls. Fall efficacy scale is reliable method to find out the fear of fall among elderly people. Reliability estimate at $0.94 .^{23}$

4. Performance oriented mobility assessment: this scale is task-oriented test that measures and older adult's gait and balance abilities by an ordinal scale of 0 to 2 .

Reliability estimated at $0.28-0.51 .^{20}$

\section{RESULTS}

In this study Group A ( $\mathrm{n}=20)$ Group $B(n=20)$ were taken with the age group of 60-80 years Male and female geriatric population with balance problem was included in the study. Result analysis has done using graphed software and paired ttest and unpaired t-test was used to comparing value within the group and between the groups.

When inter group comparison was done in BBS, TUG, and POMA there was significant in both the groups $(\mathrm{p}<0.05)$ but group A tai chi exercises were more statistically significant than group B that is stepping exercise group. When inter group comparison was done in FES, there was no significant difference found. (Table 1)

In the intra-group evaluations, changes that occurred in the values recorded before the treatment, after 2 weeks of treatment and post treatment, i.e. after 4 weeks, there was significant difference in all the parameters (BBS, TUG, FES and POMA) evaluated in both the groups ( $\mathrm{p}<$ $0.05)$

Table 1: Comparison of mean, standard deviation of post assessment values of various outcome measures between group A and group $B$ using unpaired $t$ test.

\begin{tabular}{|l|l|l|l|l|l|l|}
\hline \multirow{2}{*}{ Outcome measures } & Group A & Group B & \multirow{2}{*}{ t value } & \multirow{2}{*}{ df } & \multirow{2}{*}{ p value } & \multirow{2}{*}{ Significance } \\
\cline { 2 - 7 } & Mean \pm s.d. & Mean \pm s.d. & & & \\
\hline BBS & $42.7 \pm 2.81$ & $39.5 \pm 1.82$ & 4.272 & 38 & 0.0001 & Significant \\
\hline TUG & $8.41 \pm 0.48$ & $9.05 \pm 0.38$ & 4.63 & 38 & 0.0001 & Significant \\
\hline FES & $47.45 \pm 2.42$ & $48.8 \pm 2.12$ & 1.879 & 38 & 0.0679 & Not Significant \\
\hline POMA & $25.15 \pm 1.23$ & $24.1 \pm 0.91$ & 3.074 & 38 & 0.0039 & Significant \\
\hline
\end{tabular}

\section{DISCUSSION}

The present study was conducted to compare the effects of Tai Chi and Stepping exercise for balance training in geriatric population. Total number of 40 participants were divided into group A $(n=20)$ and group $\mathrm{B}(\mathrm{n}=20)$ respectively. The treatment was 5 sessions per week for 4 weeks each session for 45-60 minutes. Pre, intermediate and post values were taken for motor function with the independence level.

This preliminary study showed that both Tai Chi exercise and Stepping exercises induced significant improvements in balance improvement in geriatric population. At the end of training, participants randomized to either Tai Chi exercise or Stepping exercises, showed significant changes in the clinical outcomes such as BBS, TUG, FES, POMA without between-group differences.

The efficacy of Tai Chi exercise for balance improvement in geriatric population has been already documented by studies showing significant therapeutic results. Low S. et al said that most of the studies support the value of BBS for measuring recovery of balance in geriatric population. ${ }^{9}$ A study done by Fuzhong et al shows improvements in BBS score after Tai Chi exercise intervention. It also showed that Tai Chi exercise improves a physiological system that is visual, vestibular, somatosensory and musculoskeletal and neuromuscular systems are involved in postural control, thus stabilizing body positions and reducing the risk of falls. It also improves dynamic weight transition between double- stance 
and single-stance postures, constant exchange between loading and unloading of both the legs, interchange of roles between stabilizers and movers, and co-ordination between upper-body and lower-body movements. This all mechanisms contributes to postural stability in the participants which are performing Tai Chi. As postural stability increases, it improves the balance thus reducing the risk of falls. ${ }^{12}$

Another study done by Hosseini L, et al supports the values of TUG scale for recovery of balance problem in geriatric population. ${ }^{18}$ When the comparison between group A and group B was done at the post intervention, results were significant, but Tai Chi exercise was comparatively more effective than stepping exercise. The study done by Sarah Clary et al on effects of step aerobics and walking on balance in elderly shows that stepping exercise increases the strength of lower limb muscles and endurance. ${ }^{17}$ This study demonstrates that stepping exercise has a positive effect in elderly population and it improves balance, speed and co-ordination in elderly by improving and maintaining musculoskeletal strength. ${ }^{16}$

Lack of balance capacity, decrease in muscle strength, lack of joint position sense, flexibility, and the changes in gait are some of the major risk factors that are associated with falls. ${ }^{6}$ Tai Chi exercise emphasis continuous slow movement, with small to large knee movements, and rotation of the trunk, head and extremities. Tai chi exercise improves balance by improving ankle joint proprioception and foot tactile sense thus reducing the risk factors of falling. ${ }^{9}$ Tai chi also facilitates proper breathing pattern and kinesthetic awareness. This kinesthetic awareness decreases the fear of fall. Thus, Tai Chi exercises has effects on balance training. ${ }^{15}$

Another study done by Liu $\mathrm{H}$. showed that Tai chi exercise combines the physical components of the body to stay upright that is leg strength, flexibility, range of motion, speed and co-ordination; all of which tend to reduce with age. Tai Chi exercise helps in shifting of body weight properly. It also helps in improving lower limb strength. ${ }^{13}$ Tai chi exercise is smooth and coordinated movements. Hence it helps in improving the lower limb strength and coordination between both the limbs. As strength of the muscles improved, speed and range of motion also increases. Thus, Tai chi exercise is effective in balance improvement.

As expected, all the outcomes (BBS, TUG, FES and POMA) were significantly improved after treatment, irrespectively of Tai Chi exercises or stepping exercises. Tai Chi and stepping exercise both have positive effect in improving balance in geriatric population. But according to our study Tai Chi is more effective than stepping exercise in reducing the fear of fall and improving balance in geriatric population. Stepping exercise improve mostly muscle strength, flexibility and range of motion. ${ }^{16}$ Whereas Tai Chi exercise helps in improving muscle strength, flexibility, endurance, proprioception and kinesthesia which are important factors for improving balance and reducing the fear of fall in geriatric population. ${ }^{12}$

\section{Limitations of Study}

1. Sample size was less so this study can be done with large sample size.

2. The study was conducted on a smaller scale and at local level i.e. a small geographical area

3. As the Tai chi intervention was done on geriatric population, it was difficult for them to perform as they used to get exhaust very early. More research should be done on this area.

4. For long term effects, the duration of intervention should be increased.

\section{CONCLUSION}

This study provides evidence of positive effects of Tai Chi exercise and stepping exercise for improving balance in geriatric population after 4 weeks of treatment. But Tai Chi is better as compared to stepping exercise to improve balance in 
Gaurav C. Mhaske et.al. Effect of tai chi and stepping exercise for balance training in geriatrics: randomized control trial.

geriatric population. Tai Chi exercise improves neuromuscular function in our body, thus improving balance.

\section{Future Scope}

Further studies with larger sample size are required to confirm the similar results.

\section{Acknowledgement: None}

\section{Conflict of Interest: None}

\section{Source of Funding: None}

\section{Ethical Approval: Approved}

\section{REFERENCES}

1. Youngson. R, Robert M. Ageing. : Collins dictionary of Medicine; 2004,2005.

2. Borha H, Shukla P. Jain K., Kumar S. Elderly population in India. Elderly in India Profile and program2016; 24.

3. Borha H, Shukla P. Jain K., Kumar S. Gender distribution in India. Elderly in India Profile and program2016; 42

4. Royal college of physicians. Falls in older people assessing risk and prevention United Kingdom: National Institute for Health and care excellence; June 2013.

5. Faisal W., Beattie L,,Hua F., James K et al. Prevention of falls, World Health Organization, 20 Avenue Appia, 1211 Geneva 27, Switzerland: WHO Global report on Fall prevention in old age; 2017.

6. Dhargave P., Sendhilkumar R. Prevalence of risk factors for falls among elderly people living in long term care homes. Journal of clinical Gerontology and Geriatrics September 2016; 7(3).

7. Howe $\mathrm{T}$, Rochester L, Skelton D, Baallinger C. Exercise For improving Balance In Older People. 9 November 2011; 11

8. Timothy C., Fuller L., Weil L., Kotsias J., Effect of Tai Chi on Balance. Arch Otolaryngol Head Neck Surg. 1999;125:1191-1195.

9. Low S., Ang L., K. Goh. A Systemic Review of the Effectiveness Of Tai Chi On Fall Reduction Among Elderly.
Archives Of Gerontology And Geriatrics 2009; 48(3):

10. Hsiao ET, Robinovitch SN. Biomechanical influences on balance recovery by stepping. J Biomech. 1999 Oct; 32(10):

11. Wikipedia. Step aerobics. Aerobics August 2017

12. Fuzhong L, Fisher K. Harmer P. Shirai M. A Simple Eight Form Easy Tai Chi for Elderly Adult. Journal Of Aging And Physical Activity 2003; 11

13. Liu H., Frank A. Tai chi as a balance improvement exercise for older adults: a systematic review. Journal of geriatric physical therapy $2010 \mathrm{Jul}-\mathrm{sep} ; 33(3): 103-9$

14. Voukelatos A., Cumming R., et al. A randomized, controlled trial of tai chu for prevention of falls: The central Sydney tai chi control. American journal of geriatric society. 2007 Aug;55(8):1185-91.

15. Wolf S., Coogler C., T.Xu Exploring the basis for Tai Chi Chuan as a therapeutic exercise approach Journal of Physical Therapy 1997 April;77(4)371-377

16. Clary S., Barnes C., Bembem M. Effects of ballates, step exercise on balance aged 50-75 years. Journal of Sports medicine. 2006 Sep 1;5(3);390-9.

17. Oliveira M, Silva R., Dascal J. et al.: Effect of different types of exercise on postural balance in elderly women: A randomized controlled trial. Arch Gerontol Geriatric. 2014 NovDec;59(3):506-514.

18. Zeeuwe P.,The effect of Tai Chi in reducing falls among elderly people: Design of a randomized clinical trial in the Netherlands.2006 March 30;6(6).

19. Mary E., Performance oriented assessment of mobility problems in Elderly Patients. Journal of American Geriatric society. Feb 1986;34(2).

20. Mia Conra dsson, et al, Berg Balance Scale: Intrarater Test-retest Reliability Among Older People dependent in Activities of Daily Living and Living in Residential Care Facilities, Physical Therapy September 2007,87(9)1155-1163.

21. Shamway cook, predicting the probability for fall in community dwelling older 
Gaurav C. Mhaske et.al. Effect of tai chi and stepping exercise for balance training in geriatrics: randomized control trial.

adults using time up and go test, Physical Therapy (2000),80(9)896-903.

22. Schepens S., Relationship between fallRelated Efficacy and Activity Engagement in Community- Dwelling Older Adults: A Meta-Analytic Review, American journal of occupational Therapy 2012;66(2):137-14.

23. Morgan M., Friscia L., Whitney S., Furman J., et al. Reliability and Validity of the Falls Efficacy Scale-International
(FES-I) in Individuals with Dizziness and Imbalance, Otol Neurotol Aug 3013;34(6):1104-1108.

How to cite this article: Mhaske GC, Fursule D, Khairnar A. Effect of tai chi and stepping exercise for balance training in geriatrics: randomized control trial. Int J Health Sci Res. 2021; 11(6): 31-38. DOI: https://doi.org/10. 52403/ijhsr.20210606 